\title{
ESTUDIO DE LA EXTRACCIÓN DE LA GELATINA DE LA PIEL DE LA POTA
}

\author{
STUDY OF THE REMOVAL OF SKIN GELATIN OF POTA
}

\author{
Quiterio Asunción Valencia Mecola ${ }^{\mathrm{i}}$; Reyna Calcino Angulo
}

\begin{abstract}
RESUMEN
Esta investigación trata sobre la obtención de gelatina a partir de la piel de pota (Dosidicus gigas). Esta constituye entre 3 al 4 por cierto del animal y como desperdicio contamina el ambiente y debido a su contenido de proteinas colagénicas, se debe utilizar para la elaboración de cuero, harina, pegamentos y gelatina, motivo de este trabajo. El análisis proximal de la piel de la pota arroja el 81,41 de humedad; 1,058 de grasa; 13,034 de proteina y 1,016 de sales minerales. Referente a la optimización

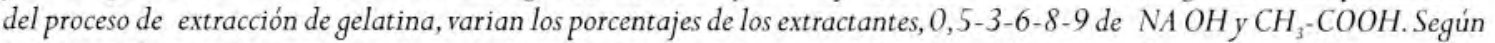
la tabla se demuestra que la óptima es de 6 a 8 por ciento y la cantidad de gelatina obtenida es de $50,2-65,9 \mathrm{~g} / \mathrm{Kg}$ piel de pota, qué es el límite de adición de estos insumos químicos, pues al aumentar se deterioran y no son aptos para consumo alimenticio. Finalmente se realizó el análisis proximal de la gelatina obtenida, el cual tiene 70,0 de proteína; 0,92 de grasa; 12,38 de humedad; 16,70 de sales minerales.
\end{abstract}

Palabras clave: Piel de pota, estroma, colágeno, gelatina, sustancias extractantes.

\section{ABSTRACT}

This research focuses on obtaining gelatin from the skin of squid (Dosidicus gigas), which is between 3 to $4 \%$ of the animal and is currently wasted, polluting the environment, and we believe it should be used for its collagenous protein content, to produce leather, flour, glue and gelatin, which is covered by this paper. The proximate analysis of the skin of the squid found that the humidity is 81.41 ; fat is 1,058; protein is 13,034 and mineral salts is 1,016, as for the optimization of gelatin extraction process, vary the percentages of extractants $\mathrm{NA}$ 0,5-3-6-8-9 $\mathrm{CH} 3 \mathrm{OH}$ and - $\mathrm{COOH}$ and a blank, according to the tables it shows that the optimum is 6 to $8 \%$ and that the amount of gelatin obtained is $50,2-65,9 \mathrm{~g} / \mathrm{kg}$ squid skin and that's the limit of adding these chemical inputs since increasing deteriorate and are not suitable for food consumption. Finally the analysis was performed proximal gelatin obtained which has 70.0 Protein, Fat 0.92 Moisture 12.38, 16.70 Mineral salts

Keywords: Squidskin, stroma, collagen, gelatine, extractants

\section{I.- INTRODUCCIÓN}

La pota o calamar gigante (Dosidicus gigas) es un cefalópodo perteneciente a las especies pelágicas oceánicas, que se encuentra en el Océano Pacifico desde California (México) hasta Cabo de Hornos (Chile) [1], concentrándose mayormente en las costas de Perú y México. $\left[{ }^{5}\right]$

Nombre científico:( Dosidicus gigas).

Nombre Común: Pota, Calamar gigante, Jibia.

Nombre en Inglés: Jumbo flying squid.

Las capturas se realizan a lo largo de nuestro litoral, por el norte, desde Tumbes hasta Pimentel- Lambayeque [7] y, por el sur, desde Mollendo hasta Tacna (estadísticas de la OROP del Pacífico).
Según la OROP $[9]$ la biomasa de la Pota en alta mar es estimada entre 2 a 4 millones de toneladas [8] y para la zona jurisdiccional en aguas peruanas, el IMARPE determinó entre 2.51 y 2.96 millones de toneladas de biomasa, con una captura permisible, en el 2012 , de 854,859 toneladas. No obstante, el máximo de captura en los últimos años fue alrededor de los 500,000 toneladas, lo cual muestra un gran margen para la expansión de la pesquería tanto en aguas de jurisdicción nacional como en la alta mar, a fin de alcanzar una explotación sostenible en beneficio económico, social y nutricional de nuestras poblaciones.

Después de la captura la pota inmediatamente es seccionada de sus partes, se corta la cabeza conjuntamente con los tentáculos, se saca las vísceras, se hace un enjuague con agua de mar, se

\footnotetext{
Recibido: 23 de octubre del 2013; Aprobado:30de octubre del 2013.

Ingeniero Pesquero, Magíster en Tecnologías de Alimentos de origen Marino, Doctor en Ciencias Naturales. Facultad de Ciencias Agropecuarias de la UNJBG.

Químico.
} 
retira las aletas y, en alguno de los casos, también la piel.

Tabla $\mathbf{N}^{\circ}$ 01. Composición física de la pota

\begin{tabular}{|l|c|}
\hline \multicolumn{1}{|c|}{ Contenido } & Promedio (\%) \\
\hline Cuerpo o tubo & 49,3 \\
\hline Aletas & 13,4 \\
\hline Tentáculos & 21,4 \\
\hline Vísceras & 15,4 \\
\hline
\end{tabular}

La piel que cubre el manto o cuerpo en promedio está constituida por el $4,66 \%$.

Fuente: Compendio biológico tecnológico de las principales especies hidrobiológicas. [t]

Actualmente se viene aprovechando los tentáculos, las aletas y el manto, pero no la piel la cual está constituida por la parte externa llamada epidermis que es de color oscuro y la parte interna o dermis que está pegada íntimamente al músculo, llamada telilla, de color blanquecina y muy resistente. La epidermis contiene cromatóforos, de los cuales, los más importantes son los melanóforos que dan coloración negro o pardo obscuro. [12]

Generalmente las pieles son arrojadas al mar conjuntamente con las vísceras y cuando se separa en la planta es arrojada a los rellenos sanitarios, contaminando, de este modo, el ambiente, sin considerar que la piel es un material orgánico que contiene proteínas que podrían dársele un valor agregado, para ser utilizado en diversas formas, por ejemplo, se podría elaborar harina de piel de pota, en todo caso, se podría curtirla y con ello elaborar diversos enseres como carteras, zapatos etc. También se puede obtener gelatina a partir del colágeno, el cual es el constituyente principal de las pieles de los animales, entre ellos, de la pota. Este es el punto de interés para nosotros y proponemos el presente trabajo de investigación.

La piel de la pota así como de la otros animales está constituida principalmente por el estroma el cual aglomera una serie de proteínas insolubles que viene a ser un grupo importante de poli péptidos y conforman el tejido conjuntivo fuerte de los tendones, de los huesos y de las capas más rígidas que envuelven y soportan a los músculos [11]

En los animales terrestres este grupo de compuestos representan aproximadamente el $35 \%$ de las proteínas de un animal vivo. [s]
En cuanto a la pota, se ha reportado que del total de la proteína presente en este organismo, el $15-18 \%$ es colágeno. Observándose que la concentración de colágeno y las propiedades térmicas dependen de la región anatómica de donde se extrae ésta proteína, siendo los tentáculos los que presentan la mayor concentración de colágeno. [16]

La gelatina es una proteína derivada de la hidrólisis del colágeno que tiene aplicaciones en alimentos, farmacia y adhesivos. [11],[15]

Hay dos procesos de producción de la gelatina, el ácido y el básico.

En el primero se trata de huesos y piel en una solución acida diluida por un período determinado, se lava con agua fría y se genera un producto con un punto isoeléctrico de 6 a 9 .

En el proceso alcalino, los huesos desmineralizados se suspenden en una solución de sosa por un periodo de 60 días; en tanto las pieles se remojan por tiempos menores, periódicamente se cambian de solución de remojo y al final se lava exhaustivamente para eliminar la sosa residual.

El punto isoeléctrico del producto está entre 4,8 y 5,2 . Tras cualquiera de esos dos procesos se extrae la proteína con agua a temperatura controlada, menor a $80{ }^{\mathrm{a}} \mathrm{C}$. En esta etapa ocurre una alteración de la triple hélice $\mathrm{D}$, en la que se rompe enlaces intermoleculares e intramoleculares y se produce cadenas menores estructuradas que corresponden propiamente a la gelatina.

Cuando el colágeno se calienta en exceso a alta temperatura se obtiene un producto amorfo y sin ninguna ordenación, que se usa como pegamento y comúnmente se llama cola. Luego de este proceso se obtiene una solución proteínica diluida $4,7 \%$, la cual se filtra, desmineraliza y se concentra en evaporadores de múltiple efecto y al vacío hasta llegar a una concentración del 25 al $30 \%$. Se esteriliza con el procedimiento de alta temperatura por corto tiempo, se enfría, se extruye y se seca. Tras cualquiera de estos casos se extrae la proteína con agua a temperatura controlada menor a $80^{\circ} \mathrm{C}$.

La vigilancia de la calidad microbiológica, durante el proceso, es crucial para el producto final, pues frecuentemente se encuentran microorganismos con actividad proteolítica que la deterioran. [Q] 


\section{OBJETIVO}

\section{Objetivo General}

- Extraer gelatina a partir de la piel de pota o calamar gigante (Dosidicus gigas).

\section{Objetivo Específicos}

- Buscar la tecnología adecuada para la extracción de gelatina a partir de la piel de la pota.

- Optimización de la tecnología de extracción de la piel de la pota.

\section{II.- MATERIALES Y MÉTODOS}

El presente trabajo es de tipo exploratorio y experimental. El material a investigar se recolectó en la planta de procesos del CEPROTEP de la UNJBG, proveniente de la zona del litoral tacneño. Se tomó una cantidad aproximada de 50 a $60 \mathrm{Kg}$ de piel, la cual fue debidamente lavada, escurrida y, enseguida, se sometió a congelación en bloques de $10 \mathrm{Kg}$ para hacer las pruebas de obtención de gelatina cada mes.

Es importante notar que la piel utilizada fue la epidermis.

\subsection{Metodología}

\subsubsection{Caracterización de la piel de la pota}

- Consistió en determinar la composición química proximal (Humedad, grasa, proteínas y las cualidades físicas de la piel según la AOAC. [1]

\subsubsection{Extracción de la gelatina de la piel de la pota}

- El proceso utilizado tiene como referencia al método desarrollado por Kolodziejska. [s]

- El trabajo realizado se muestra en el diagrama de flujo (ver Gráfico $\mathrm{N}^{\circ} \mathrm{O} 1$ ).

De acuerdo al diagrama, la piel fresca fue lavada con agua corriente, se dejó escurrir, luego se tomó $1 \mathrm{Kg}$ de piel limpia, seguidamente se procedió a pesar y moler la piel con ayuda de un molino de carne y una balanza de precisión.

En un recipiente se puso la piel molida y se añadió un 1lt de solución de $\mathrm{Na} \mathrm{OH}$, homogenizándose por primera vez, durante 15 minutos, luego de lo cual se procedió a separar el licor, el cual fue desechado.

El material se neutralizó con un 1 litro de solución de $\mathrm{CH}_{s-} \mathrm{COOH}$. y también se homogenizó durante 15 minutos. Para después hacer filtrar, hasta que desaparezca el licor.

Seguidamente se enjuagó con un 1 litro de solución de $\mathrm{NaCl}$ de una concentración $0,8 \mathrm{M}$ y se enjuagó 3 veces, empleando 3 minutos cada vez, hasta desechar el licor filtrado.

Finalmente, se lavó con agua destilada dos veces agitando durante 30 minutos y filtrando 2 veces y se desechó el filtrado.

\section{-Extracción}

Con 1 lt. de agua destilada se realizó la extracción a $70^{\circ} \mathrm{C}$ por 90 minutos por tres veces. Una vez filtrado, se guardó el licor para su clasificación.

\section{-Clarificación}

El licor resultante se trató con tierra de diatomeas al $1 \%$ a $60^{\circ} \mathrm{C}$, durante 60 minutos. Una vez filtrado se trató en carbón activado al $1,5 \%$, a $60{ }^{\circ} \mathrm{C}$., durante 30 minuto. Finalmente se filtró el licor con el papel Whatman $\mathrm{N}^{\circ} \mathrm{O} 2$.

Después el licor se colocó en unas bandejas de acero inoxidable y se sometió a desecación por aire forzado a una temperatura de $40^{\circ} \mathrm{C}$ por espacio de 24 horas.

Luego se separaron las láminas de gelatina y se pesan para establecer su rendimiento, luego se pulverizó para utilizarlas en la preparación de gelatina.

Considerando que el $\mathrm{NaOH}$ y $\mathrm{CH}_{3}-\mathrm{COOH}$ sirven para remover la estructura del colágeno y para optimizar el proceso de la gelatina hemos usado las siguientes variantes, en la primera adición de $\mathrm{NaOH}$ en la primera vez se utilizo 0,5\% y se neutralizó con $\mathrm{CH}_{3}-\mathrm{COOH}$ al $5 \%$; en la segunda vez se utilizó $\mathrm{Na} \mathrm{OH}$ al $3 \%$ y para neutralizar $\mathrm{CH}_{3}-\mathrm{COOH}$ al $3 \%$; la tercera vez se usó $\mathrm{Na} \mathrm{OH}$ al $6 \%$ y para neutralizar se uso $\mathrm{CH}_{3}$ $\mathrm{COOH}$; también al $6 \%$; la siguiente vez se uso $\mathrm{Na}$ $\mathrm{OH}$ al $8 \%$ y también $\mathrm{CH}_{3}-\mathrm{COOH}$ al $8 \%$.

2.1.3.Para finalizar se realizó el análisis proximal de la gelatina 


\section{DIAGRAMA DE FLUJO DE LA OBTENCIÓN DE GELATINA}

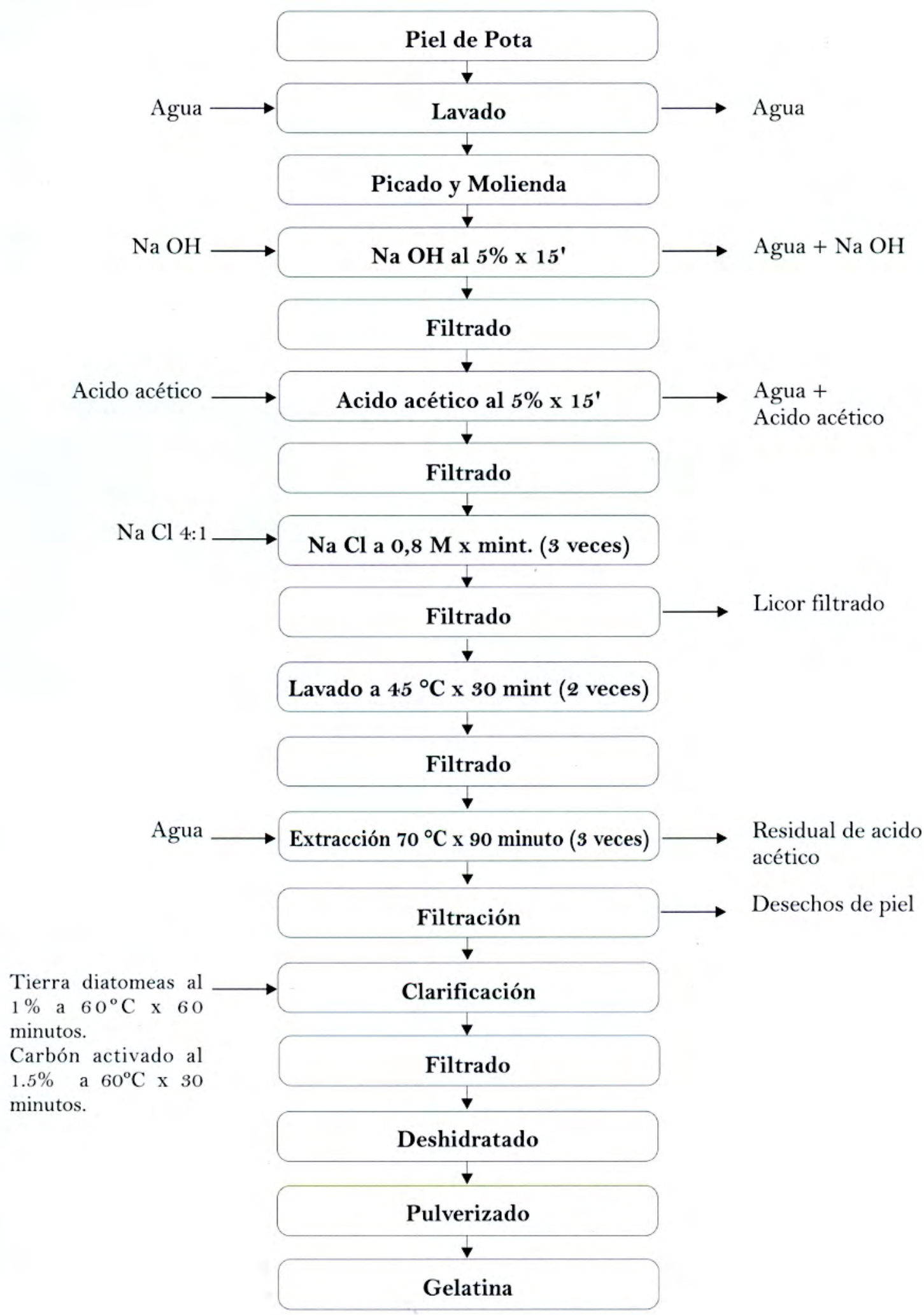




\section{III.- RESULTADOS}

Tabla $\mathbf{N}^{\mathbf{0}} \mathbf{0 2}$. Análisis proximal de la piel de Pota $(\%)$

\begin{tabular}{|c|r|r|r|r|}
\hline$N^{\circ}$ Análisis & Humedad & Grasa & Proteínas & Cenizas \\
\hline 1 & 82,73 & 1,01 & 13,99 & 1,17 \\
\hline 2 & 82 & 0,8 & 13,03 & 0,98 \\
\hline 3 & 81,4 & 1,3 & 12,95 & 1,01 \\
\hline 4 & 80,9 & 1,2 & 12,5 & 0,9 \\
\hline 5 & 80 & 0,98 & 12,7 & 1,02 \\
\hline Promedio & 81,41 & 1,058 & 13,034 & 1,016 \\
\hline
\end{tabular}

En primer lugar se determinó las características químicas de la piel de la pota cuyos resultados se muestran en el cuadro precedente

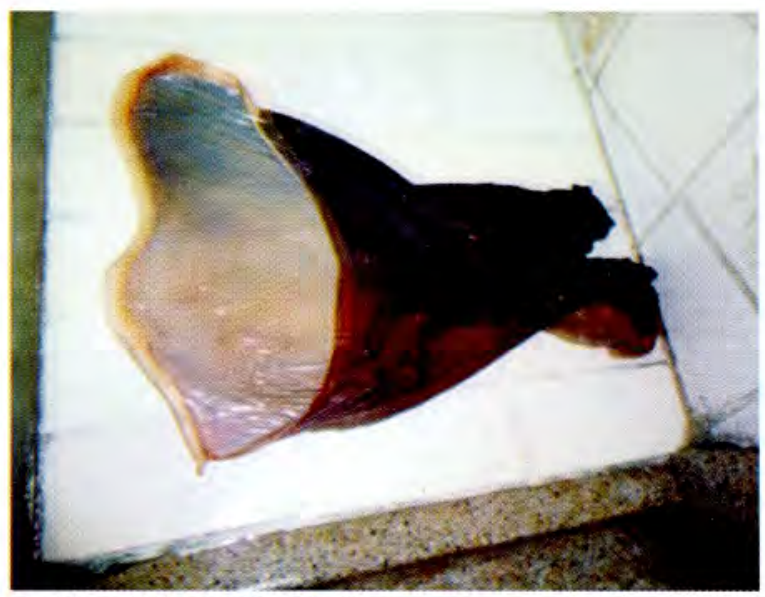

Gráfico $\mathrm{N}^{\circ}$ 02. Piel de Pota donde se aprecia que la humedad oscila entre $80-$ $82,73 \%$ y cuyo promedio es $81,41 \%$; respecto a la grasa oscila entre $0,8-1,3 \%$, resultando como promedio $1,058 \%$. En cuanto a proteínas fue de 12,5 a $13,99 \%$, promediando en $13,034 \%$ y por último las cenizas oscilaron de $0,9-1,17 \%$ teniendo como promedio 1,016.\%

Las cualidades físicas fueron determinadas organolépticamente a las cuales no se dio ninguna dimensión sin embargo, de acuerdo a nuestra percepción, la piel es de color oscuro y va variando de negro a pardo violáceo y cuando está deteriorada va tendiendo al color marrón claro, la textura es firme, elástica y bastante resistente a la tensión.

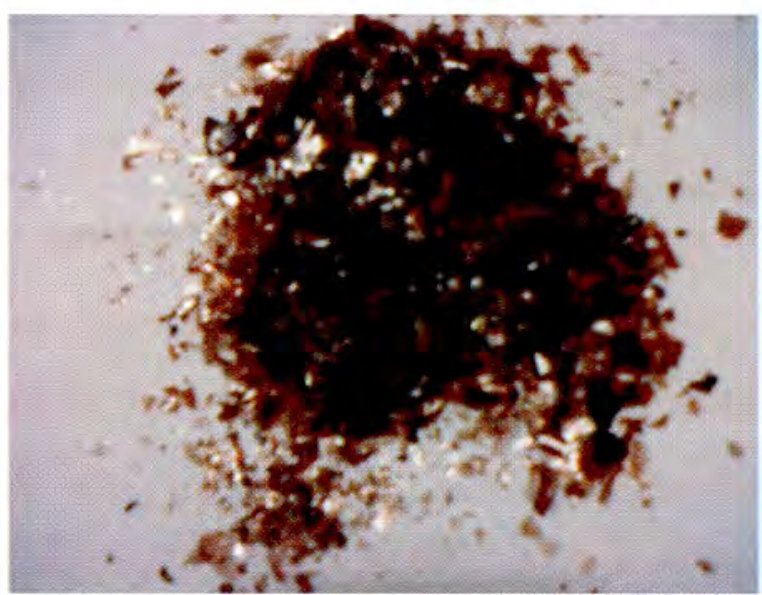

Gráfico $\mathrm{N}^{\circ}$ 03. Hojuelas de gelatina de piel de pota

Tabla $\mathbf{N}^{\circ}$ 03. Optimización de extracción de gelatina de piel de pota

\begin{tabular}{|c|c|c|c|c|c|}
\hline \multirow{2}{*}{ Mst. blanco } & $\mathrm{NaOH} \mathrm{0,5 \%}$ & $\mathrm{NaOH} 3 \%$ & $\mathrm{NaOH} 6 \%$ & $\mathrm{NaOH} 8 \%$ & $\mathrm{NaOH} 9 \%$ \\
\cline { 2 - 6 } & $\mathrm{CH} 3-\mathrm{COOH} 0,5 \%$ & $\mathrm{CH}-\mathrm{COOH} 3 \%$ & $\mathrm{CH}-\mathrm{COOH} 6 \%$ & $\mathrm{CH3}-\mathrm{COOH} 8 \%$ & CH3- $\mathrm{COOH} 9 \%$ \\
\hline 0,35 & 0,45 & 3,23 & 4,85 & 6,83 & Olor amoniacal \\
\hline 0,32 & 0,39 & 3,05 & 5,05 & 6,21 & Olor amoniacal \\
\hline 0,38 & 0,49 & 3,83 & 5,15 & 6,73 & Olor amoniacal \\
\hline Prom 0,35 & 0,44 & 3,37 & 5,02 & 6,59 & \\
\hline
\end{tabular}

En la Tabla $\mathrm{N}^{\circ}$ o3 se muestra los resultados obtenidos de la extracción de la gelatina usando los porcentajes de la sustancias que permiten la remoción de la gelatina donde se observa que el menor porcentaje obtenido corresponde al extracto hecho solamente con agua (muestra en blanco) y la gelatina extraída con la adición de $0,5 \%$ de $\mathrm{NaOH}$ y de 0,5 de $\mathrm{CH}_{3} \mathrm{COOH}$ fue ligeramente superior $(0,44 \%)$ yendo en aumento las cantidades de gelatina extraída con el aumento de los porcentajes, tanto de álcali como de ácido hasta, que llegamos al extracto obtenido con el $8 \%$, donde se logró $6,59 \%$ de gelatina y en el $9 \%$ de los componentes extractables se pudo apreciar que tenía un olor amoniacal, por lo cual ya no se tomó en cuenta.

Una vez optimizado el proceso de extracción de gelatina de piel de pota con el producto óptimo, se hizo el análisis proximal de la gelatina y por triplicado. Los resultados se muestran en la Tabla $\mathrm{N}^{\circ}$ O4 siguiente. 
Tabla $\mathrm{N}^{\circ} \mathbf{0 4}$. Análisis proximal de la gelatina de piel de pota

\begin{tabular}{|l|r|}
\hline \multicolumn{1}{|c|}{ COMPONENTE } & (\%) \\
\hline Proteína & 70,00 \\
\hline Grasa & 0,92 \\
\hline Humedad & 12,38 \\
\hline Cenizas & 16,70 \\
\hline PROMEDIO & 100,00 \\
\hline
\end{tabular}

\section{IV.- DISCUSIÓN}

El desarrollo del presente trabajo se ha hecho según el diagrama general del proceso de extracción de la gelatina de la pota, variando en porcentaje tanto de $\mathrm{NaOH}$ como de $\mathrm{CH}_{3}-\mathrm{COOH}$, tal como se indica en la metodología.

Para saber el valor del material con el que se trabajó, primeramente se hizo el análisis proximal de la piel de la pota, cuyos resultados se muestran en la Tabla $\mathrm{N}^{\circ}$ 01. Se analizó cada vez que se tenía que extraer gelatina (aproximadamente cada 45 días), las pruebas se hicieron por triplicado, lo que se muestra en la Tabla $\mathrm{N}^{\circ} \mathrm{O} 2$, con los datos los promedios de cada vez. Es importante resaltar que las muestras se tomaron en el mes de mayo del 2011 y se guardaron en congelación, sacándose cada vez que se tenía que trabajar según como se había planificado.

Los resultados obtenidos varían consecutivamente, así tenemos que la humedad de la piel fresca fue de $82,7 \%$; en cambio al finalizar el trabajo alcanzo $80 \%$. La variación fue de casi 3 puntos, lo cual es bastante significativo, probablemente se debe a la desecación que sufrió la muestra en congelación, por efecto de la circulación del aire seco en la cámara de almacenamiento y también porque las muestras estaban en la superficie del almacén o por el efecto de la desnaturalización de las proteínas o por la permeabilidad del envase de polietileno, respecto a los otros componentes como la grasa. Aquí se nota un ligero aumento a la desecación que sufrió el material, aunque esto no parece ser de mucha significancia. Respecto a las proteínas tampoco hubo mucha variación a pesar de una notoria desnaturalización de las proteínas por efecto de la sublimación del agua y la aglomeración proteica.

La optimización de la extracción se aprecia en la Tabla $\mathrm{N}^{\circ}$ 03. En la primera columna se ofrece los resultados de la muestra en blanco, la cual es como un medio de control. La extracción aquí fue invariable, se obtuvo $0,35 \%$ o sea $3,5 \mathrm{~g}$ de gelatina por $\mathrm{kg}$ de piel.
En la segunda columna están los resultados de la obtención de gelatina a partir de la piel de pota picada con la adición de una solución de $\mathrm{NaOH}$ al $0,5 \%$ y solución de $\mathrm{CH}_{5}-\mathrm{COOH}$ al $0,5 \%$, los resultados de la extracción es ligeramente mayor que la muestra en blanco donde en promedio aumentó un decimal, o sea se obtuvo $0,4 \mathrm{~g}$ de gelatina / Kg de piel. Mucho mejor es la extracción con la solución de $\mathrm{NA} \mathrm{OH}$ al aumentar los porcentajes, así como también el de elevar el del de $\mathrm{CH}_{s}-\mathrm{COOH}$; así por ejemplo cuando se llega a la adición de ambas soluciones al $8 \%$, se obtiene $6,59 \mathrm{~g}$ de gelatina / por $\mathrm{Kg}$ de piel de pota. Sin embargo no se puede seguir aumentando las concentraciones tanto de $\mathrm{NaOH}$ como de $\mathrm{CH}_{3}-$ $\mathrm{COOH}$ por cuanto el exceso trae consecuencias funestas como es el caso donde se adicionó el 9\% de $\mathrm{NaOH}$ e igual porcentaje de $\mathrm{CH}_{s}-\mathrm{COOH}$, el que dio un extracto aparentemente con mayor viscosidad pero con un olor amoniacal, lo que indicaba una hidrólisis manifiesta en el producto, hace que no sea apto para el consumo [12]. Al evaluar las gelatinas obtenidas con los diferentes porcentajes se pudo apreciar que las obtenidas con la adición de menor cantidad de aditivos fueron las mejores por su brillantez, la gelificación es mejor. Por lo que se considera que la muestra óptima para obtener la gelatina está entre 6 al $8 \%$ de $\mathrm{NAOH}$ y $\mathrm{CH}_{3}-\mathrm{COOH}$.

Respecto al análisis proximal de la gelatina de pota, según la Tabla $\mathrm{N}^{\circ}$ 04, se aprecia que el contenido proteico es elevado, lo cual se debe a la concentración mayormente de las proteínas del colágeno; sin embargo se observa que hay un exceso de sales minerales, esto se debe al remanente de material inorgánico que quedaron en el proceso de lavado de extracción de la gelatina y la formación de algunas otras sales por la adición de hidróxido de sodio y, además, por la concentración de las sales orinales que tiene la piel.

\section{V.-CONCLUSIONES}

- Podemos concluir que la composición química proximal de piel de la pota investigada en $\%$ es: Humedad 81,41, Grasa 1,058, Proteínas 13,034 y Sales minerales 1,016. Por lo cual este material puede ser utilizado en la elaboración de diversos productos tales como, cuero, harina, pegamentos y gelatina.

- Se encontró que la extracción óptima de la gelatina de la piel de pota fue con la adición de $6-8 \%$ de $\mathrm{NA} \mathrm{OH}$ y $\mathrm{CH}_{3}-\mathrm{COOH}$, con lo que se obtuvo un rendimiento de 5,02- $6,59 \%$ de gelatina o sea $50,2-65,9 \mathrm{~g}$ de gelatina / $\mathrm{Kg}$ piel de pota. 
- La composición química proximal de la gelatina optimizada es de Proteína 70,0; Grasa 0,92; Humedad 12,38 y Sales minerales 16,70.

Este trabajo sirve para clarificar muchos aspectos desconocidos con respecto a la obtención de gelatina a base de la piel de pota, así como para ver el comportamiento de la materia prima. El método utilizado nos parece satisfactorio toda vez que ha permitido obtener un rendimiento adecuado en la extracción de la gelatina.

\section{VI.- REFERENCIAS BIBLIOGRÁFICAS}

[1] Association of Official Analytical Chemists AOAC. Official methods of analysis, 15 th ed. Washington D.C., 1990.

[2] Bjarnason, A. B. 1989. Squid Fishing. INFOFISH Intl. 2(89):38-40.

[3] De Clerck, E. De Vos, P.(2002) Study of the bacterial load and the gelatine production process focussed on Bacillus and related endospore forming genera. Syst. Appl. Microbiol. Dicc; 25(4): 611-7.

[4] García Muñoz, Sandra Gpe. Mariana Perales Martínez, Luis Arturo Segura Hernández . Bioquimica de los Alimentos (2012)

[5] Gormez-Guillen, M. C., Turnay, J., Fernandez-Diaz, M. D., Ulmo, N., Lizaarbe, M. A., and Montero, P. 2002. Structural and physical properties of gelatin extracted from different marine species: A comparative study. Journal of Food Hydrocolloids 16: 2534 .

[6] Instituto del Mar del Perú-Instituto Tecnológico Pesquero del Perú. Compendio biológico tecnológico de las principales especies hidrobiológicas comerciales del Perú (marzo de 1996)

[7] Kolodziejska.L; Kaczorowski,K; Piotrowska. B, AND Sadowska.M (2004) Modification of the propierties of gelatin fron skin of Baltic. Cod (Gadus morhua) with transgluminase Food Chemistry. 70: 71-76.

[8] Morales-Bojórquez, E.; Hernández-Herrera, A.; Nevárez-Martínez, M.O.; Díaz De León, J.; ivera, G.I. \& Ramos, A. 1997. Abundancia poblacional del calamar gigante (Dosidicus gigas) en las costas de Sonora, México. Oceánides, 12: 90
[9] Maza-Ramírez, S.. Tecnología de Procesamiento de Pota "Dosidicus gigas". 2001

[10] Nigmatullin, CM, KN Nesis y AI Arkhipkin 2001. Una revisión de la biología del calamar gigante Dosidicus gigas(Cephalopoda: Ommastrephidae). Investigaciones Pesqueras54.(1)

[11] Nelson David L., Cox Micahel M. Principios de la Bioquimica de Lehninger Quinta edición.2011

[12] OROP (Organización Regional de Ordenamiento Pesquero del Pacífico Sur) (Perú Pesquero-2013).

[13] Regand, A., Goff. H.D.( 2002). Effect of biopolymers on structure and ice recrystallization in dymanically frozen ice cream model sytem. J. Datry Set.2002, Noviembre: 85 (14):2722-32

[14] Salvador Baldui Dergal. Quimica de los Alimentos IV edición-2006-pag 215

[15]Torres Marina. AOAC. Internacional Departamento de Desarrollo de Métodos.

[16] Sarabia A.l. Gomez Guillen, M.C. and Montero, $\mathrm{P}(2000$. The effect of added salts on the viscoelastic propierties of fish skin gelatin. Food Chemistry. 70: 71-76. The effect of added salts on the viscoelastic propierties of fish skin gelatin. Food Chemistry. 70: 71-76.

[17] Torres-Arreola, W., R. Pacheco-Aguilar, R. R. Sotelo-Mundo, O. Rouzaud-Sandez, y J. M. Ezquerra-Brauer. 2008. Partial Characterization of Collagen from Mantle, Fin, and Arms of Jumbo Squid (Dosidicus Gigas). Ciencia Y Tecnologia Alimentaria 6 (2):101-108.

[18] Zwiorek K, Kloeckner J, Wagner E, C Coester (2005) Gelatina nanopartículas como un sistema nuevo y simple de administración de genes. Departamento de Farmacia y Tecnología Farmacéutica Biofarmacia, Ludwig-MaximiliansUniversity, Butenandtstr. 5-13, Munich, Alemania. J Pharm Sci. Febrero $3: 7(4: 22=28)$ 
[19] Perú Pesquero del 18 de febrero del 2013pag. 09-OROP del Pacifico Sur fija cuotas de Jurel que no beneficia a Perú: La Pota pronto.

[20] Curso OCW de Eco fisiología Animal. Práctica $5^{\text {a }}$ ocw.um.es/ciencias/eco fisiologia- animal/practicas.

\section{Correspondencia}

Quiterio Valencia Mecola

Ciudad Universitaria fundo "Los Granados" Av. Miraflores s/n Tacna - Perú

Reyna Calcino Angulo

Ciudad Universitaria fundo "Los Granados" Av. Miraflores s/n Tacna - Perú
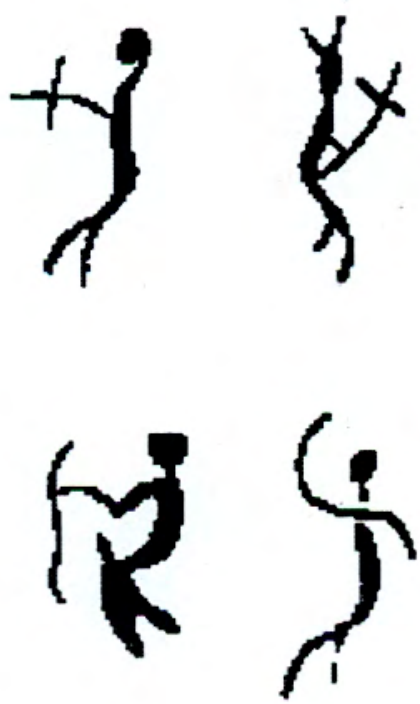